\title{
On Geometries in Affine Plane
}

\author{
Abdullah Kurudirek ${ }^{1}$, Hüseyin Akça ${ }^{1}$, Mehmet Erdoğan ${ }^{2}$ \\ ${ }^{1}$ Department of Mathematics Education, Ishik University, Arbil, Iraq \\ ${ }^{2}$ Suleyman Demirel University, Almaty, Kazakhstan
}

\section{Email address:}

drcebir@hotmail.com(A. Kurudirek), huseyinakcha@gmail.com(H. Akça), mehmet1000@yahoo.com(M. Erdoğan)

\section{To cite this article:}

Abdullah Kurudirek, Hüseyin Akça, Mehmet Erdoğan. On Geometries in Affine Plane. Applied and Computational Mathematics. Vol. 2, No. 6, 2013, pp. 127-129. doi: 10.11648/j.acm.20130206.13

\begin{abstract}
So far, in different articles and books the concepts of modern definition of geometry and Minkowskian, Galilean planes and spaces have been introduced. In this paper, we are going to describe geometry that is improved by W. Thurston and then we are going to introduce you to geometries that are suitable to this description in 2 dimensional planes.
\end{abstract}

Keywords: Non-Euclidean Geometry, Isometric, Galilean Geometry, Minkowskian Geometry, Affine Plane

\section{Introduction}

Geometry, a subject with which we are all familiar from high school, investigates properties of figures in the plane or in space. We know that the study of geometry goes back at least four thousand years, as far back as the Babylonians (2000 to $1600 \mathrm{BC}$ ). Their geometry was empirical, and limited to those properties physically observable. Through their measurements they approximated the ratio of the circumference of a circle to its diameter to be 3 , an error of less than five percent. They had knowledge of the Pythagorean Theorem, perhaps the most widely known of all geometric relationships, a full millennium prior to the birth of Pythagoras. The Egyptians (about 1800 BC) had accurately determined the volume of the frustum of a square pyramid. It is not surprising that a formula relating to such an object should be discovered by their society. Axiomatic geometry made its debut with the Greeks in the sixth century BC, who insisted that statements be derived by logic and reasoning rather than trial and error. This systematization manifested itself in the creation of several texts attempting to encompass the entire body of known geometry, culminating in the thirteen volume Elements by Euclid (300 BC). Though not the first geometry text, Euclid's Elements were sufficiently comprehensive to render superfluous all that came before it, earning Euclid the historical role of the father of all geometers. Today, the lay-person is familiar with only two, if any, names in geometry, Pythagoras, due the accessibility and utility of the theorem bearing his name, and Euclid, because the geometry studied by every high school student has been labeled "Euclidean Geometry". As is well known, already in the $17^{\text {th }}$ century the famous French mathematic cians R. Descartes (1596-1650) and P. Fermat (1601-1665) showed that Euclidean geometry can be based on the concept of number and reduced to analysis by the introduction of a coordinate system in the plane. According to Klein, geometry is the study of invariant properties of figures, i.e., properties unchanged under all motions. Geometry is a science that is based on human dream and his seeing the world, also recognizing and putting forward his idea. This science's name is formed from Greek words geo- "earth", metry- "measurement" Geometry that has a history of more than 25 century, has its own staple basis in the book of Euclid's 'Elements'. As is well known, at the beginnings of $19^{\text {th }}$ century axiomatic which is named as 'non-Euclidean' and studied by N. I. Lobachevsky caused the science to be rejuvenated, improved and new. Today, the improvement of geometry occurred by 3 dimensional manifold geometry classification that is formed by Thurston [1].

In this paper, we are going to describe geometry that is improved by W. Thurston and then we are going to introduce you with some of geometries that are suitable to this description in 2 dimensional planes[2], [3], [4] and [5].

Let $X$ be any sum and $A, B, \ldots$ are the elements of $X$. We can define the elements of $X$-sum as points. We can add the concept 'the distance between two points' to the $X-$ sum.

The function $S(A, B)$ is called the distance between the two points $A, B$ and it satisfies the condition

$$
S(A, B)=S(B, A)
$$


$(X, S)$ is the notation that can be got by adding to the distance $S(A, B)$ to the $X$-sum. We are given the sums $(X, S)$ and $\left(Y, S^{*}\right)$. The function $f$ corresponds with any value between the sums $(X, S)$ and $\left(Y, S^{*}\right)$. Thus, $f:(X, S) \rightarrow\left(Y, S^{*}\right)$.

\subsection{Definition}

If $S(A, B)=S^{*}\left(A^{\prime}, B^{\prime}\right) \quad$ for any $A, B \in X \quad$ and $A^{\prime}, B^{\prime} \in Y$ where $A^{\prime}=f(A)$ and,$B^{\prime}=f(B)$ then $f$ is called isometric transformation and it is denoted by $Y=I \operatorname{som} X$. Let's have a look at $(X, I \operatorname{som} X)$ which is the isometric sum of $X$ - sum and all $Y=I \operatorname{som} X$ that is isometric to $X$-sum.

\subsection{Definition}

The science that teaches the properties of the elements of the sum $(X, \operatorname{Isom} X)$ is called geometry.

We will show geometry that is thought in schools is parallel to the definition given above.

Let $R_{2}$ - plane and $O x y$-decart coordinate system on this plane are given. In this case, the distance between the points $A\left(x_{1}, y_{1}\right)$ and $B\left(x_{2}, y_{2}\right)$ is calculated as follows:

$$
\rho(A, B)=d=\sqrt{\left(x_{2}-x_{1}\right)^{2}+\left(y_{2}-y_{1}\right)^{2}}
$$

In formula, $R_{2}$ - plane points are shown as $X$, any other plane points are shown as $Y$. If $O^{\prime} x^{\prime} y^{\prime}$-decart coordinate system is given on $Y$ plane, and if this distance is calculated by the formula (1),

$$
f:\left\{\begin{array}{l}
x^{\prime}=x \cos \alpha-y \sin \alpha+a \\
y^{\prime}=x \sin \alpha+y \cos \alpha+b
\end{array}\right.
$$

The $f$ transformation shows that that these planes are isometric. As is well known, the transformation formula (2) gives motion in the plane. This motion consists of moving its coordinates to $C(a, b)$ - vector in a parallel way and rotate the coordinates' arrows as the measure of angle $\alpha$.

Thus, planimetry has its own place in this definition, and this is Euclidean geometry. Does any different geometry exist which is parallel to the definition given above other than Euclidean geometry? This question will be answered by our opinion given below.

Let's take the points in $A_{2}$-affine plane at $X$-sum. If the distance between the two points $A\left(x_{1}, y_{1}\right)$ and $B\left(x_{2}, y_{2}\right)$ is calculated as below,

$$
\rho(A, B)=d=\sqrt{\left(x_{2}-x_{1}\right)^{2}-\left(y_{2}-y_{1}\right)^{2}}
$$

then the distance is preserved by the given transformation formula as follows:

$$
f:\left\{\begin{array}{l}
x^{\prime}=x \operatorname{ch} \alpha+y \operatorname{sh} \alpha+a \\
y^{\prime}=x \operatorname{sh} \alpha+y \operatorname{ch} \alpha+b
\end{array}\right.
$$

In this case, a new geometry exists on the plane and this is the geometry that corresponds to Einstein's theory of relativity. By the way, distance which is set by the formula (3) may not be concluded as positive. This distance may be abstract and zero for the points which are not coinciding.

Now, let's introduce you to the idea of distance that is seperated into pieces which is different from the idea of distance mentioned above.

Let's take the distance between the given two points $A\left(x_{1}, y_{1}\right)$ and $B\left(x_{2}, y_{2}\right)$ as;

$$
d_{1}=\left|x_{2}-x_{1}\right| \text {, if } \quad d_{1}=0 \text { then } d_{2}=\left|y_{2}-y_{1}\right| .
$$

If $d_{1}=0$ and $d_{2}=0$ then $A=B$. Therefore, our points are coinciding.

\section{Theorem}

This

$$
\left\{\begin{array}{l}
x^{\prime}=x+a \\
y^{\prime}=h x+y+b
\end{array}\right.
$$

plane preserves the distance between the two points $A$ and $B$ under one unit-valued transformation where $h$ indicates the measure of the angle.

Proof: Let the points $A\left(x_{1}, y_{1}\right)$ and $B\left(x_{2}, y_{2}\right)$ are transformed to the points $A^{\prime}\left(x_{1}^{\prime}, y_{1}^{\prime}\right)$ and $B^{\prime}\left(x_{2}^{\prime}, y_{2}^{\prime}\right)$ respectively by transformation shown as (5).

It is required to prove that $|A B|=\left|A^{\prime} B^{\prime}\right|$ is true.

In fact,

$$
\left|A^{\prime} B^{\prime}\right|=\left|x_{2}^{\prime}-x_{1}^{\prime}\right|=\left|x_{2}+a-\left(x_{1}+a\right)\right|=\left|x_{2}-x_{1}\right|=|A B| .
$$

If $x_{2}^{\prime}-x_{1}^{\prime}=0$, then

$$
\begin{aligned}
\left|A^{\prime} B^{\prime}\right| & =\left|y_{2}^{\prime}-y_{1}^{\prime}\right|=\left|h x_{2}+y_{2}+b-\left(h x_{1}+y_{1}+b\right)\right| \\
& =\left|h\left(x_{2}-x_{1}\right)+y_{2}-y_{1}\right|=\left|y_{2}-y_{1}\right|=|A B| .
\end{aligned}
$$

\section{Conclusion}

So the transformation given by (5) preserves the distance in the new appearance, that is isometric transformation exists. The geometry in affine plane is called as Galilean geometry. Consequently, we informed you about Euclidean, Minkowskian and Galilean geometries in affine plane. 


\section{References}

[1] Scott P. The geometries of 3-manifods. Bull. London Math. Soc.15, 1983, n.56, p.401-487.

[2] Vincent Hugh. Using Geometric Algebra to Interactively Model the Geometry of Euclidean and non-Euclidean Spaces. February, 2007.

[3] Артыкбаев А. Соколов Д.Д. Геометрия в целом в плоском пространстве-времени. Ташкент. Изд. «Фан». 1991 г.

[4] Artıkbayev A., Kurudirek A., Akça H. Occurrence of Galilean Geometry. Applied and Computational Mathematics Vol. 2, No. 5, 2013, pp. 115-117. doi: 10.11648/j.acm.20130205.11

[5] Yaglom, I.M. A Simple Non-Euclidean Geometry and Its Physical Basis, by Springer-Verlag New York Inc. 1979.

[6] Klein, F., Vergleichende Betrachtungen uber neure geometrische Forschungen. Gesammelte mathematische Abhandlungen, Vol. I, 1921, pp. 460-497. (English version is found in Sommerville, D. M. Y., Bibliography of Non-Euclidean Geometry, 2nd ed., Chelsea, New York, 1970.)

[7] Klein, F., "Uber die sogenannte Nicht-Euklidische Geometrie," Gesammelte Math Abh I: 254-305, 311-343, 344-350, 353-383, 1921.

[8] Klein, F., Vorlesungen iiber nicht-Euklidische Geometrie. Springer, Berlin, 1928.

[9] Ross, W. Skyler B.S. NON-EUCLIDEAN GEOMETRY University of Maine, 2000. 